\title{
INICIATIVA FIGO DE PREVENCIÓN DEL ABORTO INSEGURO: VISIÓN DESDE EL MINISTERIO DE SALUD
}

"El aborto es una decisión personal tomada habitualmente como último recurso. Además los servicios de salud tienen la obligación ética de atender de la misma forma a todas las personas que solicitan atención médica. Por lo tanto, las sociedades deben garantizar que las mujeres con complicaciones derivadas de abortos legales o ilegales sean tratadas con pleno respeto y reciban una atención médica de la misma calidad que la brindada a cualquier otra persona que busca ayuda médica". Aníbal Faúndes y José Barzelato, "El Drama del Aborto. En Busca de un Consenso" (1).

La inclusión de un tema tan relevante como el aborto inseguro en la revista de la Sociedad, permite compartir con la comunidad gineco-obstétrica nacional distintas miradas, como las señaladas en las Editoriales previas, escritas por el Dr. Aníbal Faúndes primero (2), y más recientemente por el Editor de la Revista (3).

La Organización Mundial de la Salud (1992) define el aborto inseguro como "un procedimiento para finalizar un embarazo no deseado que realizan personas que carecen del entrenamiento necesario o que se lleva a cabo en un ambiente donde se carece de un estándar médico mínimo, o ambos".

El embarazo no deseado, o no planificado, condición que predispone a la realización voluntaria de un aborto provocado, se asocia -entre muchos factores- con una falta de información de la mujer sobre su fisiología reproductiva y el no uso, o uso inadecuado, de métodos anticoncepcionales eficaces. Quienes sufren mayormente las consecuencias de los abortos inseguros son las mujeres de los sectores más pobres; las mujeres con mayores recursos, pueden acceder a abortos seguros, aunque estos sean ilegales.

El acceso a la anticoncepción moderna puede reducir pero nunca eliminar la necesidad de un aborto; dado que ningún anticonceptivo es $100 \%$ efectivo, continuará habiendo embarazos no deseados, a los cuales las mujeres pueden considerar poner término a través del aborto inducido.

A nivel internacional, el aborto inseguro es considerado una pandemia persistente y prevenible; alrededor del $13 \%$ de las muertes relacionadas con embarazos han sido atribuidas a las complicaciones de los abortos inseguros, con una cifra anual estimada de 68.000 muertes (una cada 8 minutos). Se estima que alrededor de 20 millones de los abortos inducidos anualmente -cerca de la mitad- son inseguros; $95 \%$ de estos abortos ocurre en países en vías de desarrollo (4). En América Latina y el Caribe se estima que hay más de un aborto inseguro por cada 3 nacidos vivos (OMS,1998).

En este escenario, la Federación Internacional de Ginecología y Obstetricia (FIGO) formó un Grupo de Trabajo sobre Prevención del Aborto Inseguro, que conduce nuestro destacado colega el Dr. Faúndes. Esta Iniciativa de FIGO se suma a otras múltiples intervenciones de la comunidad internacional para reducir la morbilidad y mortalidad materna asociada al aborto inseguro, en especial en el mundo en desarrollo. Dado a que esta iniciativa propone la participación conjunta de la Sociedad de Obstetricia y Ginecología local y del Ministerio de Salud de cada país participante, es mi interés aportar mi visión como referente técnico ministerial en el tema.

\section{Abordaje del aborto provocado en Chile}

Nuestro país ha acumulado en los últimos 50 años una exitosa experiencia en el abordaje de este importante problema de salud pública y de la salud de las mujeres. En 1960 el número de egresos hospitalarios por aborto alcanzaba a 57.368, de los cuales un 75 a $90 \%$ eran provocados, responsables de un 35 a $40 \%$ de las muertes maternas. La magnitud del problema llevó al Profesor Hernán Romero -Director del Departamento de Medicina Preventiva de la Universidad de Chile y presidente del Colegio Médico- a hablar de la "epidemia" del aborto provocado $(5,6)$. 
En respuesta a esta grave situación, en 1962 el Director General del Servicio Nacional de Salud (SNS), convoca un grupo asesor -la Comisión Asesora en Política de Regulación de la Fecundidad- para reducir la alta incidencia de abortos inducidos y sus complicaciones, y para reducir el alto costo asociado al tratamiento de los abortos complicados. En 1964, siguiendo la recomendación de esta Comisión Asesora, se formula la política de planificación familiar del Gobierno de Chile, la cual se incluye en las actividades de protección de la salud materno-infantil; su objetivo general era "reducir los riesgos asociados a la práctica del aborto inducido en condiciones inseguras“..., "garantizando el respeto a la conciencia de las personas y su dignidad".

La importante reducción observada en la magnitud del aborto provocado -número de egresos hospitalarios por abortos inducidos y la mortalidad materna por aborto (de 302 muertes anuales en 1960, hoy debemos lamentar no más de 4 - 5 muertes)- refleja la exitosa implementación en el país de las actividades de planificación familiar/regulación de la fertilidad. La prevalencia actual de uso de métodos anticonceptivos en la población usuaria del sistema público de salud se estima en un $60 \%$; si bien los indicadores cuantitativos son altamente satisfactorios, hay otros aspectos cualitativos en los cuales es posible avanzar.

En la actualidad la realización de cualquiera forma de aborto está completamente prohibida y penalizada en el país, luego de la eliminación del "aborto terapéutico" en Septiembre de 1989, por el Gobierno Militar; por su condición de ilegalidad y de penalización, no se dispone de cifras reales para conocer la magnitud del aborto provocado.

A través de estimaciones indirectas, a partir del número de egresos hospitalarios por complicaciones, la cifra va desde alrededor de 60.000 abortos por cada 100 mujeres de 15 a 49 años (7) a 120.000 abortos clandestinos al año (8). En el instrumento que recoge la información sobre los egresos hospitalarios (Informe Estadístico de Egreso Hospitalario), en muchos casos no se precisa la naturaleza del aborto (espontáneo, provocado). El año 2006 se registraron 23.052 egresos por aborto, y se estima que un $20 \%$ de estos abortos son espontáneos, por lo cual 18.350 de estos egresos corresponderían a complicaciones de abortos provocados.

Más allá de la cifra que más se aproxime a la realidad nacional de esta práctica, lo realmente importante es como reducir la necesidad de muchas mujeres de tomar esta decisión tan dramática, ante situaciones personales muy complejas, con sus potenciales riesgos de morbimortalidad, sea a través de la prevención del embarazo no deseado -que ha sido la política institucional que ha seguido el Ministerio desde hace casi 45 años- o, de un manejo integral adecuado, que permita reducir estos riesgos y se oriente a prevenir una recurrencia a futuro.

\section{El contexto internacional actual}

Los conceptos de Salud Sexual y de Salud Reproductiva, originalmente propuestos por la Organización Mundial de la Salud, con la activa movilización internacional de organizaciones sociales y de mujeres, fueron aprobados y ratificados a través del consenso alcanzado en diversas conferencias internacionales realizadas en los últimos 15 años: Conferencia Mundial sobre Población y DesarroIlo (El Cairo, 1994), Conferencia Mundial sobre la Mujer, (Beijing, 1995), Cumbre del Milenio (Nueva York, 2000).

Para el logro de una buena Salud Sexual y Reproductiva (SSR) en todas las personas, se ha asignado especial relevancia al reconocimiento y respeto de los derechos reproductivos y sexuales individuales, entendidos como el derecho de hombres y mujeres a decidir libremente y a tener opciones en lo concerniente a la libertad y seguridad de la persona, en el ejercicio de su sexualidad y capacidad reproductiva, el derecho a la vida, la calidad de atención en salud, el acceso a la información, y otras cuestiones como la privacidad y la confidencialidad en la toma de decisiones en el ámbito de su SSR.

Hoy se reconoce que los derechos sexuales y reproductivos son parte de los derechos humanos, derechos que el Estado tiene la responsabilidad de adoptar las medidas que sean necesarias para asegurar su ejercicio a todas las personas, sin ninguna forma de discriminación. En la atención integral de la salud de la mujer, resulta fundamental asegurar las condiciones que le permitan hacer decisiones reproductivas debidamente informadas, entregando servicios de buena calidad en los distintos sistemas y niveles de atención (público y privado).

En el marco ético formulado en 1994 por la OMS, en relación a los cuidados ginecológicos y obstétricos, se destaca el principio de autonomía (rol que la mujer debe adoptar en el cuidado de su salud). Este principio invita a tratar a cada individuo como una persona libre e independiente, capaz de tomar decisiones y aceptar la responsabilidad de ellas; sirve de base al consentimiento informado de las personas ante decisiones relacionadas con su aceptación o rechazo, de distintos procedimientos médicos.

En el caso del aborto, implica reconocer que 
cada mujer puede decidir continuar o interrumpir su embarazo. Este principio obliga al prestador de servicios de salud a guardar el secreto profesional, como parte de los derechos del paciente a la privacidad y a la confidencialidad: Comité de Derechos Humanos (ONU, 2000) "...el forzar a una mujer embarazada a llevar a término un embarazo no deseado o riesgoso para su salud, puede llevar a situaciones que constituyen un trato cruel, inhumano o degradante."

Una mujer que cursa un embarazo no deseado -independiente de lo que puedan pensar terceras personas- tiene diferentes opciones: continuar el embarazo y aceptar al recién nacido/a; aceptar el embarazo y entregar el recién nacido/a en adopción, o interrumpir el embarazo. Cuando una mujer solicita atención por aborto, sus necesidades están determinadas por sus circunstancias particulares, por diversas situaciones específicas muy complejas; por ello, resulta fundamental tener una actitud empática en su atención, centrada en los aspectos propiamente relacionados con su salud.

Como sujeto de derechos, cualquiera sea la opción que elija, la mujer tiene derecho a una atención integral en salud y por lo tanto el sistema sanitario debe disponer de una normativa que la proteja y le brinde opciones en ese momento de máxima vulnerabilidad. Los marcos de los derechos humanos y la bioética definen que estas mujeres en situación de aborto deben recibir una atención digna, humanizada y de alta calidad, evitando el maltrato innecesario al interior de los establecimientos hospitalarios (humanización). Esta ha sido una demanda concreta para nuestro sector por parte de diferentes organizaciones de la sociedad civil.

La calidad de atención en este ámbito implica apoyar y respetar la toma de decisiones informadas de las mujeres; garantizar la confidencialidad, privacidad y respeto a estas decisiones (interacción adecuada entre las mujeres y el personal de salud, respeto a los principios fundamentales de la bioética: autonomía, beneficencia, no maleficencia, justicia); en establecimientos debidamente equipados y uso de tecnologías adecuadas por personal de salud calificado (9).

\section{Modelo de reducción de riesgos y daños en salud}

La mayoría de las acciones de salud que requieren las mujeres son realizadas por ginecólogos y obstetras. En el Congreso Mundial de la especialidad (1994), el Dr. Mahmoud Fathalla presidente de FIGO, en su documento "Salud de la mujer: una mirada global", señala: "La especialidad de la Gineco-
Obstetricia ha desarrollado tecnologías que pueden salvar vidas y mejorar la salud de las mujeres; sin embargo, el impacto sobre la salud de las mujeres aún deja bastante que desear. No podemos seguir escondiendo nuestras cabezas en las arenas de la biología y dar la espalda a las realidades sociales que afectan negativamente la salud de las mujeres. Nosotros podemos ser tanto parte del problema como parte de la solución".

Los derechos a la salud y a la información están fuertemente ligados a la justicia social; el derecho a la información (Artículo 19, Declaración Universal $\mathrm{DDHH}$ ), constituye un aspecto central en la toma de decisiones y sirve de base para el ejercicio de otros derechos. La operacionalización de estos derechos constituye la base del modelo de reducción de riesgos y daños en salud, desarrollado en Uruguay por médicos gineco-obstetras del ámbito académico y asistencial, con apoyo de FIGO e IPPF (10).

Este modelo -acogido por el Ministerio de Salud Pública uruguayo se materializa en una norma de atención sanitaria a nivel nacional (11)- ha permitido abordar el problema del embarazo no deseado y el aborto inseguro en los servicios de salud, dentro del marco legal vigente, y el respeto de los derechos de las mujeres. Este modelo se ha basado en el concepto de informar, que en términos legales es diferente al de prescribir o promocionar una decisión determinada.

\section{Mejoramiento de los servicios de SSR}

Muchos de los conceptos internacionales señalados están contenidos en los principios que han desarrollado los sucesivos Gobiernos democráticos a partir de 1990 (igualdad del hombre y la mujer en todos los planos de la sociedad; autonomía de la mujer como persona capaz de tomar sus decisiones y comprometerse con la sociedad; dignidad de la mujer y la valoración de sus capacidades y su quehacer).

En 1997, el Ministerio de Salud define la Salud Sexual y Reproductiva como una de las 16 Prioridades Nacionales de Salud, y ha definido metas específicas en este ámbito como parte de los Objetivos Sanitarios para la década 2000 - 2010, y como metas accesorias en los compromisos del país en las Metas de Desarrollo del Milenio. Uno de los 3 objetivos sanitarios formulados en relación a este tema es: Reducir la incidencia del aborto provocado, a través del mejoramiento de la oferta y la calidad de los servicios de SSR (prevención primaria), con una focalización especial en la población de mayor riesgo de aborto provocado. La prevención de la recurrencia del aborto requiere de una ade- 
cuada coordinación entre los servicios hospitalarios que atienden mujeres después de un aborto y los establecimientos de atención primaria (12).

La no accesibilidad a ninguna forma de aborto, requiere ofrecer a la población servicios de SSR de la mejor calidad posible (decisión personal plenamente informada, amplia gama de métodos anticonceptivos eficaces), a través del reconocimiento y pleno respeto de los derechos reproductivos individuales; este desafío requiere de un cambio de mentalidad tanto por parte de los prestadores como de la población usuaria.

Las "Normas Nacionales sobre Regulación de la Fertilidad" (Ministerio de Salud, 2007), aprobadas por Decreto Supremo, precisan que en las actividades relacionadas con la SSR, debe respetarse el derecho a la privacidad y confidencialidad: "La preservación de la intimidad y la privacidad de los individuos, así como el favorecer la autonomía y el proceso de la toma de decisiones, resultan básicos para quienes trabajan en los temas de salud sexual y reproductiva. Por ello, la atención debe realizarse en un ambiente privado, asegurando a quien consulta que la información que entrega es confidencial" (13).

\section{Conclusión}

La tarea de elaborar un Plan de Acción que permita abordar integralmente la problemática del aborto inseguro en nuestro país, a través del trabajo conjunto entre la Sociedad Chilena de Ginecología y Obstetricia, el Ministerio de Salud y otras instituciones nacionales e internacionales, con la activa participación de grupos organizados de la sociedad civil, constituye una oportunidad para dar un nuevo paso en la protección de la salud y los derechos de las mujeres de nuestro país. El aporte de los ginecólogos y obstetras, médicos de mujeres, es funda- mental, para el desarrollo y la implementación del Plan que logremos construir en forma asociada.

Dr. René Castro S. Programa Salud de la Mujer. Ministerio de Salud de Chile.

\section{BIBLIOGRAFIA}

1. Faúndes A, Barzelato J. El Drama del Aborto. En Busca de un Consenso. Editorial Tercer Mundo (Bogota, 2005).

2. Faúndes A. La Iniciativa de FIGO para la prevención del aborto inseguro, Rev Chil Obstet Ginecol 2008;73(4):221-2.

3. Donoso E. ¿Unsafe abortion en Chile? Rev Chil Obstet Ginecol 2008;73(6):359-61.

4. OMS: Aborto sin Riesgo: Guía técnica y de política para sistemas de salud (2003).

5. Plaza S, Briones A. El Aborto como Problema Asistencial. Rev Méd Chile, 1963.

6. Armijo R. Monreal T. Epidemiología del Aborto Provocado en Santiago, Rev Méd Chile, 1964.

7. Lavín P. Informe preliminar sobre la caracterización de los casos y costos del tratamiento del aborto hospitalizado en Santiago de Chile (Bogotá, 1994).

8. Weisner M. Aborto Clandestino: Ayer, Hoy y .... ¿Mañana? VI Congreso Chileno de Antropología. Valdivia, Noviembre 2007.

9. IPAS: La atención del aborto centrada en la mujer (2006).

10. Carino G, Barroso C, Ward V. Un modelo basado en derechos: perspectivas desde los servicios de salud, (IPPF, Iniciativas Sanitarias, Uruguay, Febrero de 2008).

11. Ministerio de Salud Pública: Ordenanza 369/04 (Uruguay, Agosto de 2004).

12. Ministerio de Salud. Los Objetivos Sanitarios para la década 2000 -2010; 9-12, 2002.

13. Ministerio de Salud. Normas Nacionales sobre Regulación de la Fertilidad, 2007. 